\title{
Ocular MR Imaging as a Substitute for Ultrasound during the COVID-19 Pandemic
}

$\mathbf{T}$ he novel coronavirus disease 2019 (COVID-19) pandemic is a public health emergency of international concern, bringing a multitude of new challenges in caring for patients. There is considerable variability in the prevalence of the virus as well as the availability of personal protective equipment (PPE) worldwide.

Ultrasonography examinations involve close, often prolonged (>10 minutes) physical contact with patients and confer a high risk of viral transmission by respiratory droplets. This is particularly the case for ocular ultrasound, with the practitioner positioned directly above the patient's head in close proximity to the upper respiratory tract and nasopharnyx. ${ }^{1}$ Furthermore, Severe Acute Respiratory Syndrome coronavirus 2 (SARS-CoV-2) viral RNA has been detected in ocular swab samples in patients with conjunctivitis and in ocular fluids in the early incubation period before the onset of characteristic respiratory symptoms. Therefore, ocular fluids may not only be the point of virus entry but also a source of contagion to other patients and eye care professionals. $^{2}$

Strict protective measures by each ultrasonography operator are necessary when performing an examination on patients positive for COVID-19. The American Institute of Ultrasound in Medicine advises that all patients be considered as possibly infected with COVID-19 for the purposes of protecting personnel. ${ }^{3}$ Radiologic and ophthalmologic societies recommend that all examinations be strictly limited to an absolute minimum. Given the limited supply of PPE, health care professionals are at high risk of contamination, including those in ophthalmology, previously considered a low-risk subspecialty. ${ }^{4}$

In response to the COVID-19 pandemic, we, as an institution, have devised a high-resolution MR imaging protocol as a substitute for ocular ultrasonography in the setting of ophthalmologic emergencies. This is an initiative by the radiology department in collaboration with our ophthalmology colleagues, as a risk-mitigation strategy to protect staff while maintaining optimum patient care. ${ }^{1}$ Local institutional review board authorization was granted.

-m Indicates open access to non-subscribers at www.ajnr.org http://dx.doi.org/10.3174/ajnr.A6819
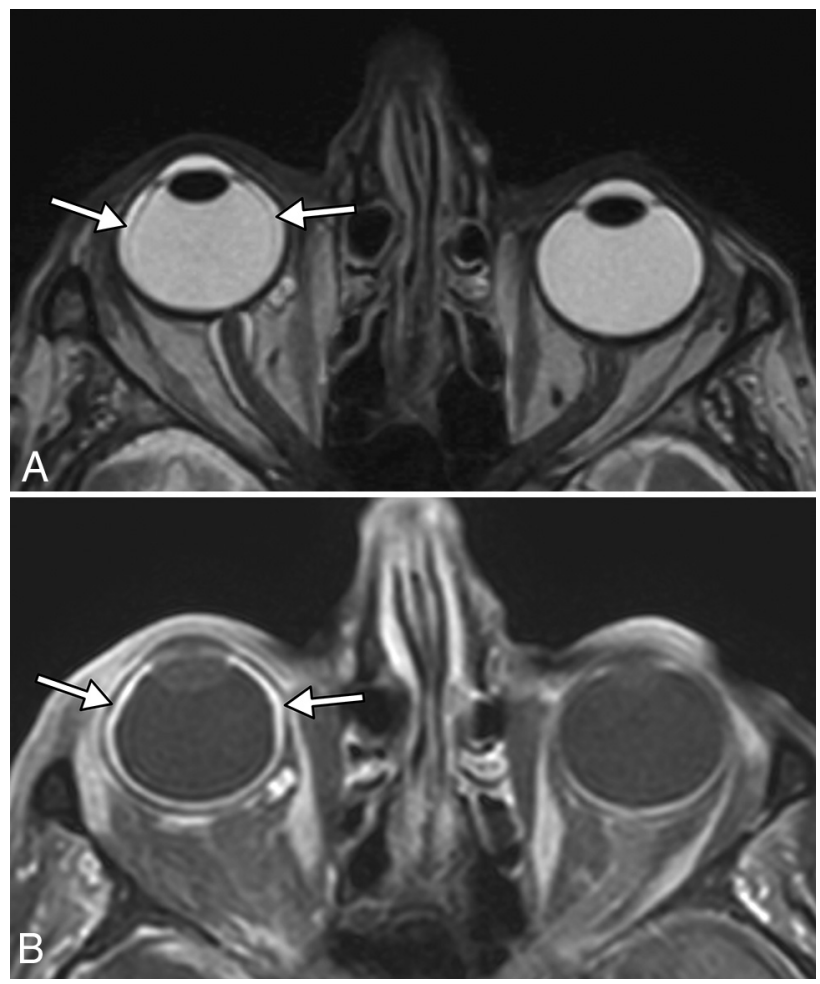

FIG 1. Axial MR imaging T2-weighted sequence $(A)$ demonstrating a thin membrane (arrows) in the right globe extending anteriorly to the ciliary body, with corresponding enhancement on postcontrast T1weighted sequence ( $B$, arrows), consistent with a thin peripheral ciliochoroidal detachment. Postcontrast imaging is useful to confirm choroidal detachment because the choroid displays frank enhancement as opposed to the retina or sclera.

All MR imaging examinations are performed at 3T. Our protocol is centered on the orbits with high-resolution 3D T1-weighted sequences before and after intravenous injection of gadolinium chelate and a 3D T2-weighted sequence. We obtain high in-plane spatial resolution of the order of $0.45-0.55 \mathrm{~mm}$, which is obtainable on most commercially available MR imaging scanners. Practically, this allows reconstructions in any plane approximating the 

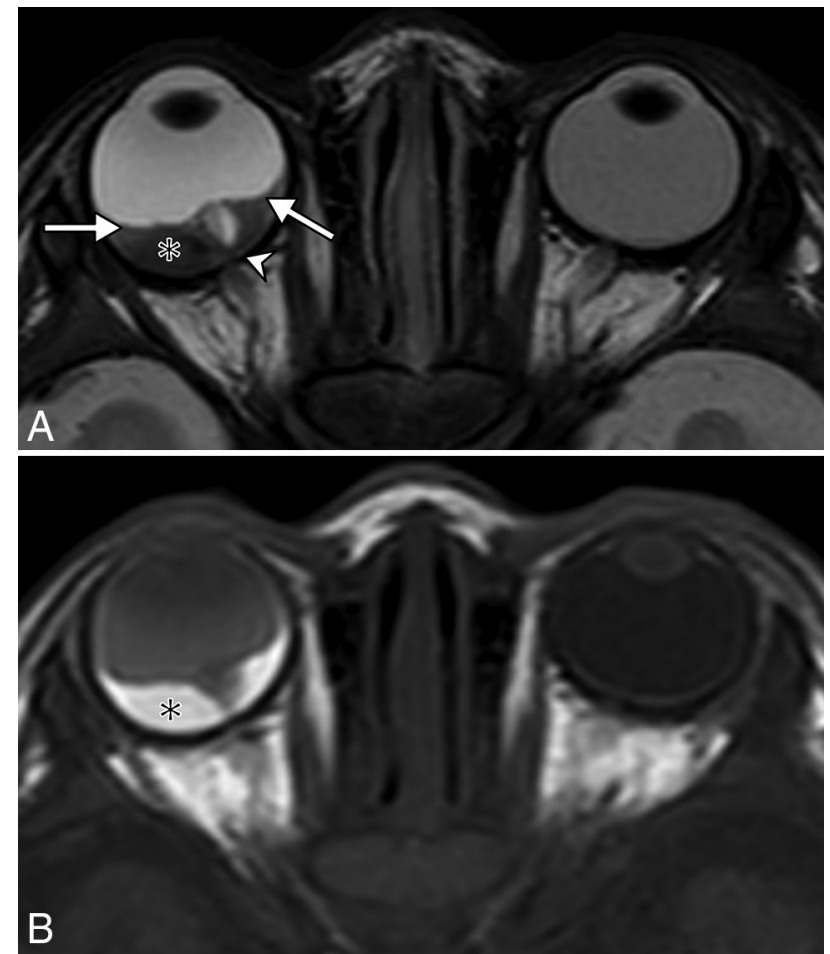

FIG 2. Axial MR imaging T2-weighted sequence $(A)$ demonstrating a $\mathrm{V}$-shaped membrane (arrows) converging on the right optic disc posteriorly (arrowhead), limited by the ora serrata anteriorly. This is the classic appearance of total retinal detachment, confirmed at surgery. There is an underlying subretinal hematoma (asterisk); these are intrinsically Tl-hyperintense (B). Note the slightly T1-hyperintense vitreous compared to the contralateral side, consistent with vitreous hemorrhage, initially seen at slitlamp examination.

accuracy of B-mode ultrasound at $10 \mathrm{MHz}$, which can localize anomalies according to standard field or meridian nomenclature.

Urgent or emergent examinations are determined on an individual basis but include those entities requiring immediate clinical or surgical intervention, often with sight-threatening complications, including ocular trauma, retinal detachment, choroidal detachment, vitreous hemorrhage, and ocular tumors. We illustrate the value of ocular MR imaging in some of these settings (Figs 1-3).

In conclusion, we show that ocular MR imaging can be a useful diagnostic tool as a substitute for ultrasound and a temporary measure during the COVID-19 pandemic. The sensitivity and specificity of MR imaging compared with ultrasound for the investigation of ocular emergencies is not currently known; therefore, further studies are required.

Disclosures: Julien Savatovsky—UNRELATED: Consultancy: Bayer AG; Payment for Lectures Including Service on Speakers Bureaus: Philips, Medtronic; Travel/ Accommodations/Meeting Expenses Unrelated to Activities Listed: Bayer AG.
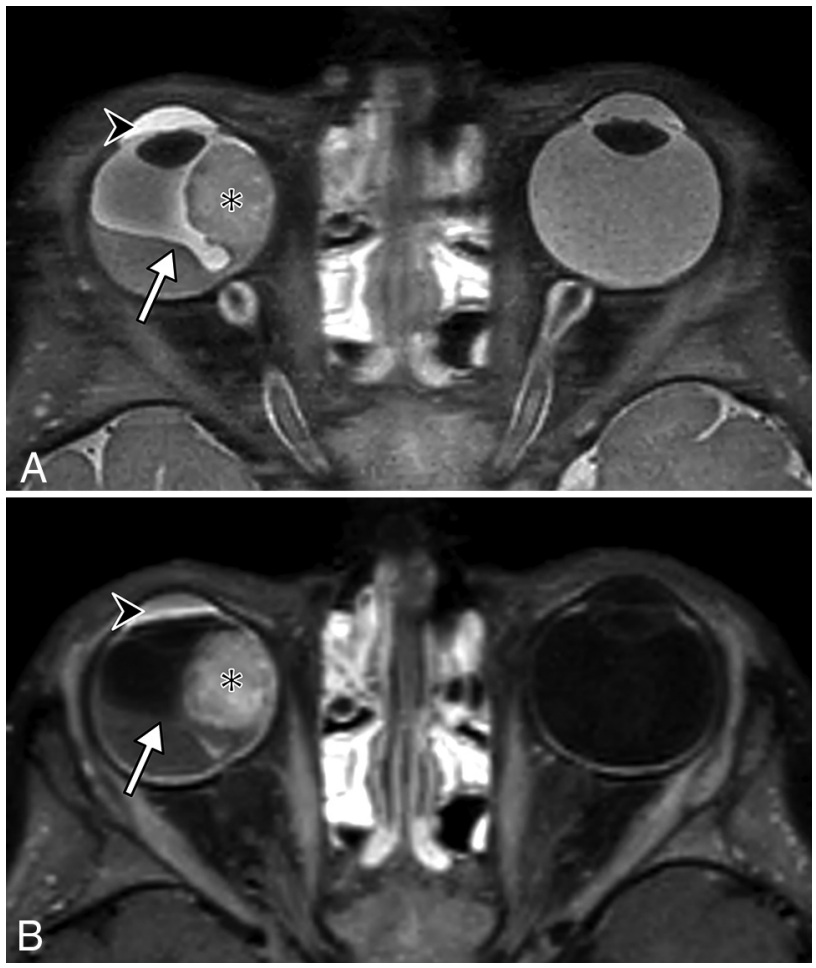

FIG 3. Axial MR imaging T2-weighted $(A)$ and T1-weighted post-IV gadolinium $(B)$ sequences demonstrating the classic V-shaped membrane of a retinal detachment (arrow), converging on the optic disc posterior to the right globe, due to an underlying, exophytic, enhancing mass in the nasal quadrant (asterisk). There are also inflammatory changes in the anterior chamber (arrowhead).

\section{REFERENCES}

1. Ji-Peng OL, Shantha J,Wong TY, et al. Preparedness among ophthalmologists: during and beyond the COVID-19 pandemic. Ophthalmology 2020;127:569-72 CrossRef Medline

2. Colavita F, Lapa D, Carletti F, et al. SARS-CoV-2 isolation from ocular secretions of a patient with COVID-19 in Italy with prolonged viral RNA detection. Ann Intern Med 2020;173:242-43 CrossRef Medline

3. American Institute of Ultrasound in Medicine. Quick Guide on COVID-19 Protections-Patient and Ultrasound Provider Protection. 2020. https://aium.s3.amazonaws.com/covid19/Covid19_Quick_Guide_ PUPP.pdf. Accessed April 24, 2020

4. Qiao C, Zhang H, He M, et al. Symptomatic COVID-19 Infection in Eye Professionals in Wuhan China. Ophthalmology 2020;127(9):12681270 CrossRef Medline 